\title{
DESIGN APPROACHES AND MATERIALS PROCESSES FOR ULTRAHIGH EFFICIENCY LATTICE MISMATCHED MULTI-JUNCTION SOLAR CELLS
}

\author{
Melissa J. Griggs ${ }^{1}$, Daniel C. Law ${ }^{2}$, Richard R. King ${ }^{2}$, Arthur C. Ackerman ${ }^{3}$, James M. Zahler ${ }^{3}$, Harry A. Atwater ${ }^{1}$ \\ ${ }^{1}$ California Institute of Technology, 1200 East California Boulevard, Pasadena, California 91125, USA \\ ${ }^{2}$ Spectrolab Engineering Inc., 12500 Gladstone Avenue, Sylmar, California 91342, USA \\ ${ }^{3}$ Aonex Corporation, 129 North Hill Avenue, Suite 108, Pasadena, California 91106, USA
}

\begin{abstract}
In this study, we report synthesis of large area $\left(>2 \mathrm{~cm}^{2}\right)$, crack-free GaAs and GalnP double heterostructures grown in a multi-junction solar cell-like structure by MOCVD. Initial solar cell data are also reported for GalnP top cells. These samples were grown on $\mathrm{Ge} / \mathrm{Si}$ templates fabricated using wafer bonding and ion implantation induced layer transfer techniques. The double heterostructures exhibit radiative emission with uniform intensity and wavelength in regions not containing interfacial bubble defects. The minority carrier lifetime of $\sim 1$ ns was estimated from photoluminescence decay measurements in both double heterostructures.

We also report on the structural characteristics of heterostructures, determined via atomic force microscopy and transmission electron microscopy, and correlate these characteristics to the spatial variation of the minority carrier lifetime.
\end{abstract}

\section{INTRODUCTION}

High efficiency triple junction solar cells have recently been produced with efficiencies exceeding $39 \%$ [1]. To achieve the highest efficiencies, the band gaps of the materials used in multi-junction solar cells must be optimized to efficiently absorb as much of the solar flux as possible. This is complicated by the additional requirement in monolithic devices that all the materials be lattice-matched. Future ultra-high efficiency multi-junction solar cells will utilize lattice-mismatched structures to achieve an optimal bandgap sequence for solar energy conversion. While lattice-mismatched multi-junction cells have been fabricated recently using metamorphic growth approaches [2], use of direct wafer bonding techniques to enable lattice mismatch accommodation at the subcell interfaces facilitates considerably more design freedom and inherently higher quality, defect-free, active regions. Our work has focused on a four-junction InGaP/GaAs/GalnAsP/InGaAs cell featuring two optimized, internally lattice-matched two-junction subcells (see Fig.1). The top two junction cell is grown on a template latticematched to GaAs, whereas the bottom two junction cell is grown on a template lattice-matched to $\mathrm{InP}$. Subcells are integrated via a direct bond interconnect between the GaAs and GalnAsP.

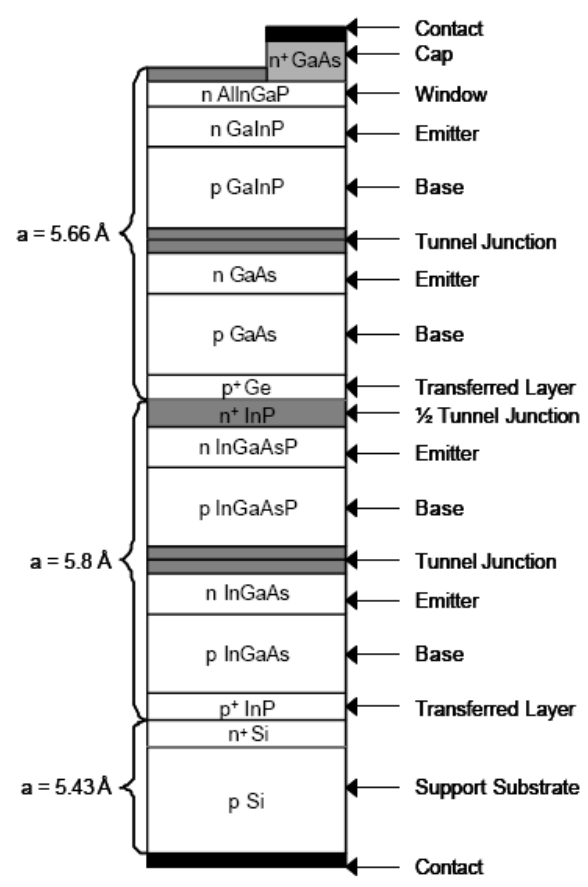

Fig. 1. Schematic of the proposed wafer bonded four-junction solar cell, where a denotes the lattice constant of the materials.

For this structure to be viable, we require ohmic contacts at the bonded interfaces (series resistance can degrade the efficiency of concentrator solar cells) and good quality epitaxial growth on bonded templates. The bonded templates are created by layer transfer and wafer bonding utilizing co-implantation of $\mathrm{H}^{+}$and $\mathrm{He}^{2+}$ for the layer splitting process. In this manner, we can carefully control the thickness of our transferred film. Astransferred, these films are rough (RMS>10nm) and they have a $\sim 200 \mathrm{~nm}$ thick damaged layer that must be removed to enable further epitaxial growth. Damage can be removed and the roughness can be abated for both the $\mathrm{Ge} / \mathrm{Si}$ and the InP/Si transferred films using a simple wet etch (see Fig. 2). In addition, the morphology of the surface roughness could affect the quality of the epitaxial films grown on the template, so we also explored the effects of Ge homoepitaxy on our transferred layers. 

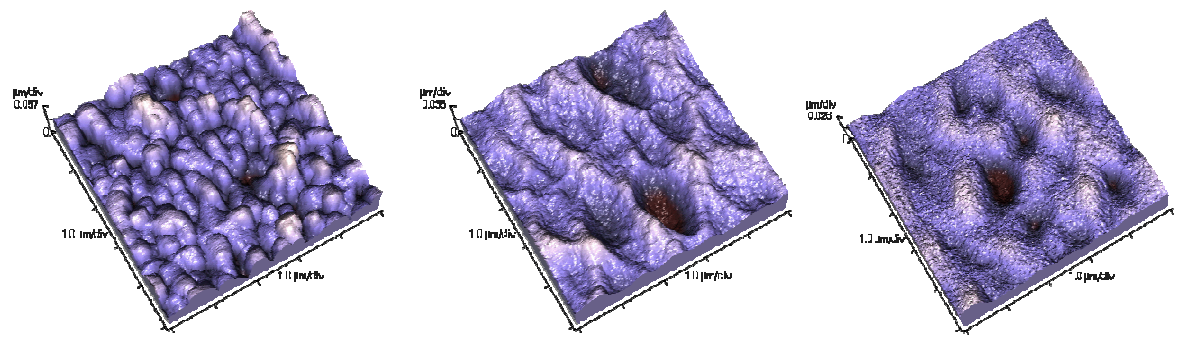

Fig. 2. $5 \mu \mathrm{m} \times 5 \mu \mathrm{m}$ AFM scans of Ge/Si template as-transferred (RMS $=20 \mathrm{~nm}$ ), after wet-etch $($ RMS $=9 \mathrm{~nm})$, after wet-etch $+200 \mathrm{~nm}$ homoepi $(\mathrm{RMS}=8 \mathrm{~nm})$.
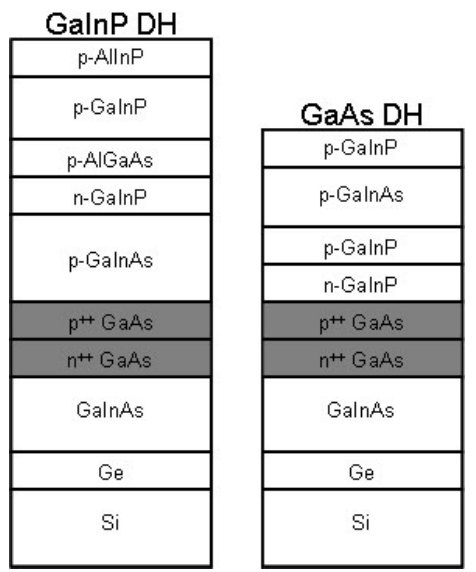

Fig. 3. Schematic of the two double heterostructures grown on $\mathrm{Ge} / \mathrm{Si}$ templates.

III-V double heterostructures were grown on these Ge/Si templates; one with an AIGaAs/GalnP/AIGaAs active region, the other with a GalnP/GaAs/GalnP active region. These will be referred to as GalnP and $\mathrm{GaAs}$ double heterostructures $(\mathrm{DH})$ respectively.

\section{EXPERIMENTAL PROCEDURES}

\section{Ge/Si Templates}

Ge/Si templates were fabricated using a coimplantation of $\mathrm{H}^{+}$and $\mathrm{He}^{2+}$ into $\mathrm{Ge}$ substrates. These implanted substrates were then hydrophobically bonded to Si substrates. At elevated temperature and pressure, the implanted $\mathrm{Ge}$ wafers split along the peak implantation position producing a Ge/Si template and a Ge donor wafer. The templates were prepared for epitaxial growth with a modified CP4 etch to remove the residual damage from the implantation. Since the coefficient of thermal expansion mismatch between $\mathrm{Ge}$ and $\mathrm{Si}$ is quite

large [3], the $\mathrm{Ge}$ donor wafers were also prepared for epitaxial growth using the same etch treatment to isolate the effects of surface morphology from those due to CTE mismatch-induced strain. Also, 200nm of Ge homoepitaxy was grown on some of the templates before III-V DH growth to enable us to study the effects of different types of surface morphologies. The homoepitaxy samples showed much rounder surface features when compared to the as-transferred and etched $\mathrm{Ge} / \mathrm{Si}$ templates. In order to better understand the effects of our damage removal etch, Ge epi-ready substrates were etched under the same conditions as the templates. Ge epi-ready wafers with no processing were used as controls in all growths. The growth structures were designed to mimic two-junction solar cell structures (see Fig. 3).

The damage removal processing was analyzed using spectroscopic ellipsometry to measure the Ge film thickness as a function of etch time. Using the implant dose, we estimated the damaged region to be $\sim 200 \mathrm{~nm}$ thick. Good quality $\mathrm{Ge}$ homoepitaxy can be achieved on our templates with a 15 s etch (see Fig. 4), thus confirming that our etch process is removing all of the damage.

\section{RESULTS}

\section{AIGaAs/GalnPIAIGaAs Double Heterostructures}

The GalnP DHs were shown to be optically active by mapping the photoluminescence (PL) over large areas of the transferred films $\left(\sim 6 \mathrm{~cm}^{2}\right)$. The PL was measured using 50mW 488nm laser excitation for all GalnP $\mathrm{DH}$ samples. The collection slit width and pump power were not changed between samples, so the PL intensity is comparable among all samples (see Fig. 5). The $\mathrm{DH}$ on the $\mathrm{Ge} / \mathrm{Si}$ template did show the lowest intensity, but it was still within an order of magnitude of the control sample.

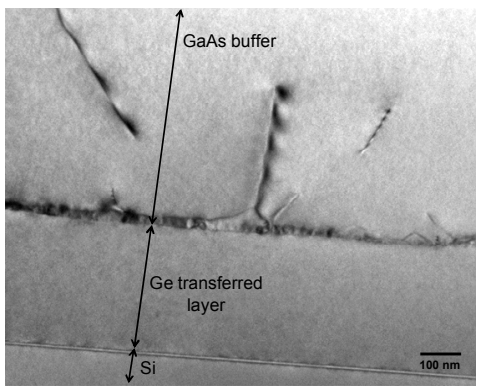

Fig. 4. Cross-sectional TEM images of III-V double heterostructures grown on Ge/Si templates.
Since the Ge donor wafer sample shows a higher intensity, the CTEmismatch is inducing noncatastrophic strain in the III-V growth. However, the surface morphology appears to be the dominant factor in the performance of 


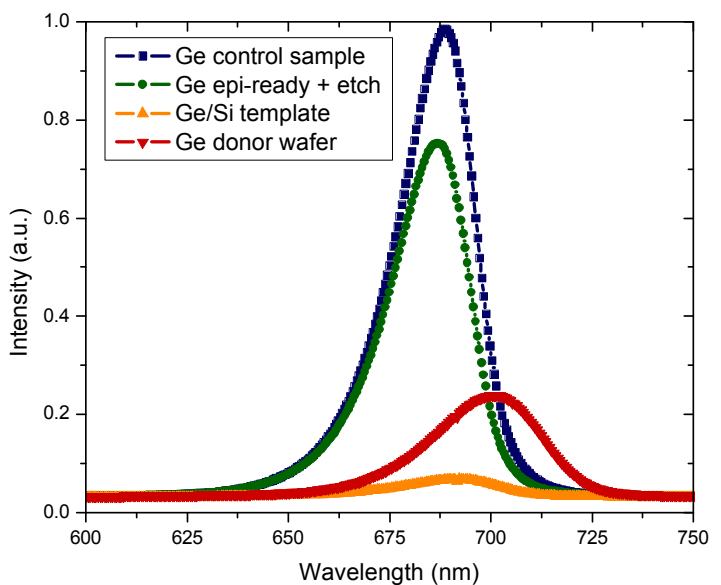

Fig. 5. Photoluminsecence spectra from GalnP active region grown on bulk epi-ready $\mathrm{Ge}$, epi-ready $\mathrm{Ge}+$ etch, $\mathrm{Ge} / \mathrm{Si}$ template, and the $\mathrm{Ge}$ donor wafer.

\begin{tabular}{|c|c|c|}
\hline DH Type & Sample & Lifetime (ns) \\
\hline GalnP & ClT Control & 4.78 \\
\hline GalnP & Epi-ready + Etch & 1.87 \\
\hline GalnP & Ge/Si Template & 0.799 \\
\hline GalnP & Ge Donor Wafer & 1.89 \\
\hline GaAs & Control & 112 \\
\hline GaAs & Epi-ready + Etch & 85.6 \\
\hline GaAs & Ge/Si Template & 0.124 \\
\hline GaAs & Ge Donor Wafer & 0.104 \\
\hline GaAs & Control (2) & 188 \\
\hline GaAs & Ge/Si Template (2) & 1.03 \\
\hline
\end{tabular}

Table 1. Photoluminsecence intensity and peak position from the GalnP active region grown on bulk epi-ready $\mathrm{Ge}$ and the $\mathrm{Ge} / \mathrm{Si}$ template.

these structures, since intensity of the donor wafer is only four times the template sample.

In order to quantitatively compare the performance of these samples, time-resolved photoluminescence (TRPL) measurements were performed on all of these samples (see Table 1). The TRPL measurements show that the template sample is of a high quality since it is only four times smaller than the control sample at $\sim 1 \mathrm{~ns}$. Again, a factor of two is gained in the $\mathrm{Ge}$ donor wafer sample, showing that the surface morphology still needs to be improved.

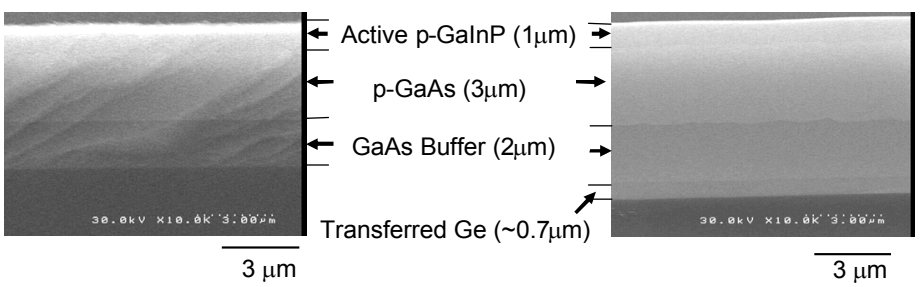

Fig. 6. Cross-sectional SEM images of GalnP active structure on epi-ready $\mathrm{Ge}$ (left) and $\mathrm{Ge} / \mathrm{Si}$ template (right).
Cross-sectional SEM and TEM were used to better understand how the templates were affecting the material structure of the DHs. The cross-sectional SEM images of these structures show a slight roughening at the interfaces, which is most apparent at the GaAs buffer/pGaAs interface (see Fig. 6). Cross-sectional TEM of these structures shows that all of the residual defects and damage from the ion implantation were successfully removed, so this is not the cause of the lower performance. In addition, the Ge homoepitaxy grown on templates is high quality with a good interface to the template layer (see Fig. 4). Therefore, the templates are useful for homoepitaxy, but the application to heteroepitaxy still needs to be improved. The miscut of the template samples may be imperfect after processing making it more difficult to grow high quality III-V materials. The GaAs buffer layer grown in the structures shows good bulk quality, though there is a high density of defects nucleating just at the surface of the template layer (see Fig. 4). Upon closer examination in high-resolution TEM, this appears to be caused by some degree of contamination on the surface, perhaps a residual oxide layer. Similar contamination can be seen on the bulk epiready $\mathrm{Ge}$ control sample. In addition, cathodoluminescence $(C L)$ measurements taken on the template samples show the defect density to be $\sim 8 \times 10^{6} \mathrm{~cm}^{-2}$, whereas the Ge donor wafer sample shows a dislocation density of $\sim 1 \times 10^{3} \mathrm{~cm}^{-2}$. The epi-ready Ge that was exposed to the same etching procedures as the template samples shows an extremely low threading dislocation density in $\mathrm{CL}\left(\sim 30 \mathrm{~cm}^{-2}\right)$, but a dense crosshatch pattern, which is typical for lattice-mismatched materials. This suggests that the surface morphology may be affecting the ordering in the GalnP, rather than just producing dislocations.

\section{GalnP/GaAs/GalnP Double Heterostructures}

The GaAs DHs were also optically active, but the peak intensity of the Ge/Si templates and the Ge donor

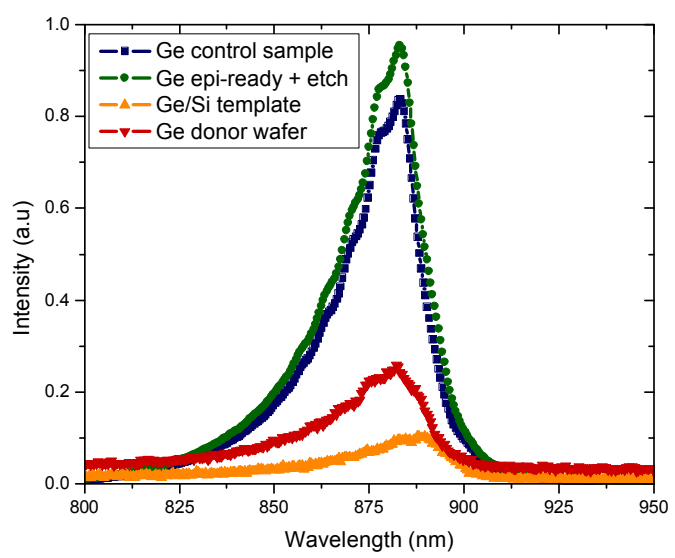

Fig. 7. Photoluminsecence spectra from GalnP active region grown on bulk epi-ready $\mathrm{Ge}$, epi-ready $\mathrm{Ge}+$ etch, $\mathrm{Ge} / \mathrm{Si}$ template, and the $\mathrm{Ge}$ donor wafer. 
wafers was significantly lower than the control samples. The pump beam was the same as in the GalnP DHs, but the collection slits were adjusted between measurement of the two epi-ready samples and the Ge/Si template and donor wafer. The peak intensities of the template and donor wafers cannot be directly compared. They have been artificially increased to allow direct comparison of the peak positions and peak shapes. The peak position of the template sample is slightly red-shifted compared to all the other samples (see Fig. 7). The CTE-mismatch appears to be causing the peak shift since it is not in the donor wafer sample. However, as with the GalnP DHs, the surface morphology appears to dominate the intensity results. TRPL measurements of these samples show, though the PL intensity is quite low, the GaAs active region on the $\mathrm{Ge} / \mathrm{Si}$ template has a lifetime of $\sim 1 \mathrm{~ns}$ (see Table 1).

\section{GalnP/GaAs Two Junction Solar Cells}

Preliminary two junction solar cells were grown on $\mathrm{Ge} / \mathrm{Si}$ templates as well as $\mathrm{Ge}$ donor wafers and $\mathrm{Ge}$ control wafers. These initial cell results are without antireflective coating and without optimizing the growth parameters. Spectral response measurements were taken on the GalnP top cell and converted to external quantum efficiency (see Fig. 8). The Ge/Si template shows about the same overall quantum efficiency as the donor wafer giving us further proof that the surface preparation is dominating the performance of these devices, not the CTE-mismatch induced strain. However, there is some red response loss in the template sample denoting a lower diffusion length in the template sample. Initial light IV data shows promise for the template samples as the short circuit current is similar to the donor wafer sample (see Fig. 9).

\section{CONCLUSIONS}

Now that we have demonstrated reasonable top cell performance on Ge/Si template samples, the next step

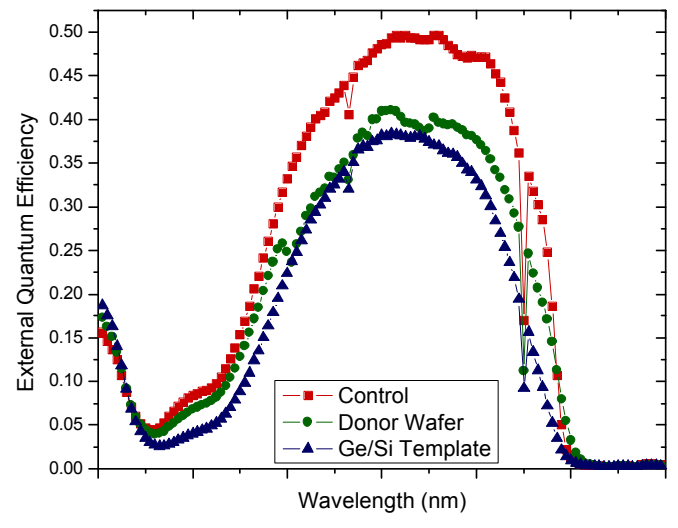

Fig. 8. External quantum efficiency of a GalnP cell grown as part of a two junction GalnP/GaAs solar cell on bulk epi-ready Ge, a Ge donor wafer, and a $\mathrm{Ge} / \mathrm{Si}$ template. is to optimize the surface preparation. Chemicalmechanical polishing techniques should further improve the surface roughness of these templates.

Our transferred films performed very well mechanically in surviving X-TEM sample prep as well as the large thermal cycles required for metalorganic chemical vapor deposition, indicating that the $\mathrm{Ge}$ is covalently bonded to the Si and the CTE-mismatch is not catastrophic for normal processing conditions. These results show promise for the use of transferred Ge layers as epitaxial templates for GaAs-based semiconductor heterostructures on Si.

\section{ACKNOWLEDGEMENTS}

The authors would like to acknowledge W. Metzger for measuring the time-resolved photoluminescence spectra, C. Garland for her assistance in the TEM work, and R. Walters for his assistance with the photoluminescence intensity measurements.

Funding for this work was provided by the National Renewable Energy Laboratory. M. Griggs acknowledges fellowship support from the National Science Foundation.

\section{REFERENCES}

[1] R. King, et al., "Metamorphic III-V Materials, Sublattice Disorder, and Multi-junction Solar Cell Approaches With Over 37\% Cell Efficiency," Proc. $19^{\text {th }}$ European Solar Cell Conf. \& Exhibition, Paris, France 2004

[2] M. Wanlass, et al., "GalnP/GaAs/GalnAs Monolithic Tandem Cells for High-Performance Solar

Concentrators ," Proc. International Conf. on Solar

Concentrators for the Generation of Electricity or Hydrogen, Scottsdale, USA 2005.

[3] L. Freund and S. Suresh, Thin Film Materials: Stress, Defect Formation and Surface Evolution, Cambridge University Press, Cambridge 2004.

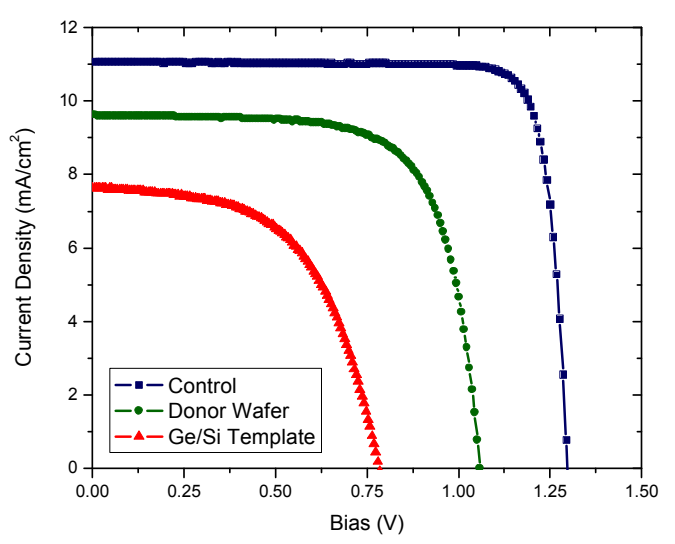

Fig. 9. Light I-V data for GalnP cells on bulk epiready $\mathrm{Ge}$, a $\mathrm{Ge}$ donor wafer, and a $\mathrm{Ge} / \mathrm{Si}$ template. 